\title{
ON SOME DIFFICULTIES IN THE THEORY OF TRANSFINITE NUMBERS AND ORDER TYPES
}

\author{
By B. Russell.
}

[Received November 24th, 1905.-Read December 14th, 1905.]

DR. Hobson's most interesting paper in the Proceedings of the London Mathematical Society* raises a number of questions which must be answered before the principles of mathematics can be considered to be at all adequately understood. I do not profess to know the complete answers to these questions, and most of the present paper will consist only of tentative suggestions, made as possibly a step towards the true solutions, not as themselves constituting solutions. With the greater part of Dr. Hobson's paper I find myself in agreement; my purpose, therefore, will not be in the main polemical, but rather to carry the discussion a stage further by introducing certain distinctions which I believe to be relevant and important, and by generalizing as far as possible the difficulties and contradictions hitherto discovered in the theory of the transfinite.

There are two wholly distinct difficulties to be considered in the theory of transfinite cardinal numbers, namely :

(1) The difficulty as to inconsistent aggregates (as they are called by Jourdain);

(2) The difficulty as to what we may call Zermelo's axiom. $\dagger$

These two difficulties do not seem to be clearly distinguished by Dr. Hobson; yet they are, so far as appears, largely independent and of very different degrees of importance. The first leads to definite contradictions, and renders all reasoning about classes and relations, prima facie, suspect; while the second merely raises a doubt as to whether a certain much used axiom is true, without showing that any fundamental difficulties arise either from supposing it true or from supposing it false. I shall consider these difficulties separately, beginning with the first, because it is more fundamental.

* Series 2, Vol. 3, pp. 170-188.

+ See his "Beweis, dass jede Menge wohlgeordnet werden kann," Math. Annalen, Vol. Irx., pp. 514-516. For statements of various forms of this axiom see the third part of this paper. 
I.

When Dr. Hobson speaks of the necessity of a norm for constituting an aggregate, he appears sometimes to suppose that the norm is absent or ill-defined in the case of inconsistent aggregates, at other times to suppose it absent where Zermelo's axiom requires it. But the two cases are, in reality, quite distinct. The doubt as to the truth of Zermelo's axiom arises from the impossibility of discovering a norm by which to select one term out of each of a set of classes, while the difficulty of inconsistent aggregates arises from the presence of a perfectly definite norm combined with the demonstrable absence of a corresponding aggregate. This suggests that a norm is a necessary but not a sufficient condition for the existence of an aggregate; if so, the complete solution of our first set of difficulties would consist in the discovery of the precise conditions which a norm must fulfil in order to define an aggregate. Logical determinateness, it seems, is not sufficient, as Dr. Hobson supposes (p. 173), and the meaning which he attaches to the term aggregate (ib.) appears to be too wide. This is proved by a perfectly strict argument, which I shall try to state after explaining some ways of generating inconsistent aggregates.

In the first place, since the discussion belongs to symbolic logic, which already possesses technical names for the ideas we require, it is desirable to compare Dr. Hobson's terms with those in current use. What he calls a norm is what I call a propositional function. A propositional function of $x$ is any expression $\phi ! x$ whose value, for every value of $x$, is a proposition ; such is " $x$ is a $\operatorname{man}$ " or " $\sin x=1$." Similarly we write $\phi !(x, y)$ for a propositional function of two variables; and so on.

In this paper I shall use the words norm, property, and propositional function as synonyms.

The word aggregate is used sometimes with an implication of order, sometimes without; I shall use class where there is no implication of order, and where there is order I shall consider the relation of before and after which generates the order. This last is necessary because every class which can be ordered at all can be ordered in many ways; so that only the ordering relation, not the class, determines what the order is to le. A relation will be used in an extensional sense, i.e., so that two relations are identical provided each holds whenever the other holds. We shall find that a propositional function $\phi ! x$ may be perfectly definite, in the sense that, for every value of $x, \phi ! x$ is determinably true or determinably false, while yet the values of $x$ for which $\phi ! x$ is true do not form a class. And, similarly, we shall find that a propositi nal function $\phi !(x, y)$ 
may be in the same sense definite, without there being any relation $R$ which holds between $x$ and $y$ when and only when $\phi !(x, y)$ is true.

In order to eliminate at the outset a number of considerable but irrelevant difficulties, I may point out that the argument $w e$ are about to consider does not depend upon this or that view as to the nature of classes and relations. The refutable assumption as to the nature of classes and relations is only this: that a class is always uniquely determined by a norm or property containing one variable, and that two norms which are not equivalent (i.e., such that, for any value of the variable, both are true or both false) do not determine the same class, with a similar assumption as regards relations. It is in no way essential to the argument to suppose that classes and relations are taken in extension, i.e., that two equivalent norms determine the same class or relation. Thus the argument proves that a norm itself is in general not an entity; that is, if we make statements of the form $\phi ! x$ about a number of different values of $x$, we cannot pick out an entity $\phi$ which is the common form of all these statements, or is the property assigned to $x$ when we state $\phi ! x$. In other words, a statement about $x$ cannot in general be analyzed into two parts, $x$ and what is said about $x$. There is no harm in talking of norms or properties so long as we remember this fact; but, if we forget it, we become involved in contradictions.

The two contradictions first discovered concerned respectively the greatest ordinal and the greatest cardinal.* Of these the cardinal contradiction is the simpler, and lends itself more readily to the removal from arithmetic to logic which I wish to effect for both. I shall therefore consider it first.

The cardinal contradiction is simply this: Cantor has a proof $t$ that there is no greatest cardinal, and yet there are properties (such as " $x=x$ ") which belong to all entities. Hence the cardinal number of entities having such a property must be the greatest of cardinal numbers. Hence a contradiction.

If every logically determinate norm defines a class, there is no escape from the conclusion that there is a cardinal number of all entities. For, in that case, the norm " $x=x$ " defines a class, which contains all entities : call this class $V$. Then the norm " $u$ is similar to $V$ " defines a set of classes which may be taken as being the cardinal number of $V$,

* The contradiction concerning the greatest ordinal was first set forth by Burali Furti, "Una questione sui numeri transfiniti," Rendiconti del Circolo Matematico di Palermo, 1S97. The contradiction concerning the greatest cardinal is discussed in my Principles of Mathematics, $\S 344 . f f$.

† Jahresbericht der Deutschen .Mathematiker-Vereinigung, Vol. I., 1892, p. 77. 
i.e., the greatest cardinal number.* Thus, if every logically determinate norm defines a class, it is impossible to escape the conclusion that there is a greatest cardinal.

The other horn of the dilemma yields more interesting results. Cantor's proof that there is no greatest cardinal may be simplified into the following:-Let $u$ be any class, and $R$ a one-one correlation of all the members of $u$ to some (or all) of the classes contained in $u$. There are such correlations, since one of them is obtained by correlating each member of $u$ with the class whose only term is that member. Consider now the following norm :- " $x$ is a member of $u$, but is not a member of the class with which $R$ correlates it." Suppose this norm defines a class $w$. Then $v$ is omitted from the correlation ; for, if $w$ were correlated with $x$, then, if $x$ is any member of $w$, it follows from the definition of $w$ that $x$ is not a member of its correlate, i.e., is not a member of $w$; while, conversely, if $x$ is not a member of $w$, it is a member of its correlate, i.e., of $w$. Hence the supposition that $w$ is the correlate of $x$ leads to a contradiction. Hence, in any one-one correlation of all the terms of $u$ with classes contained in $u$, at least one class contained in $u$ is omitted. Therefore, whatever class $u$ may be, there are more classes contained in $u$ than there are members of $u$.

We may test this conclusion, in the case of the class of all entities, by constructing, according to the method of the proof of the Schröder-Bernstein theorem, an actual one-one correlation of all terms with all classes, and then considering the class which Cantor shows to be omitted. This process leads us to the consideration of the norm: " $x$ is not a class which is a member of itself." If this norm defines a class $w$, then the class $w$ is omitted from our correlation. But it is easy to see that this norm does not define a class at all. For, if it defined a class $w$, we should find that, if $w$ is a member of itself, then it is not a member of itself, and vice versa. Hence there is no such class as $w$. Essentially the same argument may be stated as follows:-If $u$ be any class, then, when $x=u$, the statement " $x$ is not an $x$ " is equivalent to " $x$ is not a $u$." Hence, whatever class $u$ may be, there is one value of $x$-namely, $u$-for which " $x$ is not an $x$ " is equivalent to " $x$ is not a $u$ "; thus there is no class $w$ such that " $x$ is not an $x$ " is always equivalent to " $x$ is a $w . "$ Hence, again, this norm does not define a class.

We thus find that, quite apart from any view as to the nature of cardinals, and without any considerations belonging to arithmetic, we can prove that at least one perfectly determinate norm does not define a class.

- I shall consider later Dr. Hobson's objection to this definition. 
By the same method, we can easily construct other such norms. Take any class $u$ for which we can correlate all entities to some $u$ 's by a one-one correlation. By the method of the proof of the Schröder-Bernstein theorem, construct an actual one-one correlation of all the members of $u$. to all classes contained in $u$, and then consider the norm: " $x$ is a member of $u$ which is not a member of its correlate according to the correlation in question." This norm does not define a class. Thus from the class of all relations we obtain the norm: " $R$ is not a relation which is a member of its own domain." From the class of all couples we obtain the norm: " $v$ is such that the couple whose members are (1) the class of all entities, (2) $v$, is not a member of $v$." Thus it appears that the contradiction dealt with in chapter x. of my Principles of Mathematics is a special case of a general type of contradictions which result from supposing that certain propositional functions determine classes, when, in fact, they do not do so. The above method of discovering such propositional functions is not required for proving, when they are discovered, that they are of the sort that do not define classes. In each case it is easy to discover a definite simple contradiction, analogous to that discussed in the above mentioned chapter, which results from supposing that the propositional functions in question do determine classes.

In like manner propositional functions of two variables do not always determine relations. For example, " $R$ does not have the relation" $R$ to $S$ " does not determine a relation $T$ between $R$ and $S$, i.e., it is not equivalent, for all values of $R$ and $S$, to " $R$ has the relation $T$ to $S$." For, if it were, substituting $T$ for $R$ and for $S$, we should have " $T$ does not have the relation $T$ to $T$," equivalent to " $T$ has the relation $T$ to $T$," which is a contradiction.

The following contradiction, of an analogous type to those discussed above, shows that a norm or property is not always an entity which can be detached from the argument of which it is asserted. Consider the norm " $x$ does not have any property which it is." If this assigns to $x$ the property $\theta$, then " $x$ has the property $\theta$ " is equivalent to " $x$ does not have any property which it is." Hence, substituting $\theta$ for $x$, " $\theta$ has the property $\theta$ " is equivalent to " $\theta$ does not have any property which it is," which is equivalent to " $\theta$ does not have the property $\theta$ "; whence a contradiction. The solution, in this case, is that properties are not always (if ever) separable entities which can be put as arguments either to other properties or to themselves. Thus, when we speak of properties we are sometimes (if not always) employing an abbreviated form of statement, which leads to errors if we suppose that the properties we are speaking of are genuine entities. 
We have thus reached the conclusion that some norms (if not all) are not entities which can be considered independently of their arguments, and that some norms (if not all) do not define classes. Norms (containing one variable) which do not define classes I propose to call non-predicative; those which do define classes I shall call predicative. Similarly, by extension, a norm containing two variables will be called predicative if it defines a relation; in the contrary case it will be called non-predicative. Thus we need rules for deciding what norms are predicative and what are not, unless we adopt the view (which, as we shall see, has much to recommend it) that no norms are predicative.

I come now to Burali-Forti's contradiction concerning the greatest ordinal, and I shall show how this too reduces to a simple logical contradiction resulting from supposing that a certain non-predicative function is predicative.

Burali-Forti's contradiction may be stated, after some modification, as follows:-If $u$ is any segment of the series of ordinals in order of magnitude, the ordinal number of $u$ is greater than any member of $u$, and is, in fact, the immediate successor of $u$ (i.e., the limit if $u$ has no last term, or the immediate successor of the last term if $u$ has a last term). The ordinal number of $u$ is always an ordinal number, and is never a member of $u$. But now consider the whole series of ordinal numbers. This is well ordered, and therefore should have an ordinal number. This must be an ordinal number, and yet must be greater than any ordinal number. Hence it both is, and is not, an ordinal number, which is a contradiction.

To generalize this contradiction, put $\phi ! x$ in place of " $x$ is an ordinal," and $f^{\prime} u^{*}$ in place of "the ordinal number of $u$." Then in the case of the ordinals $\phi$ and $f$ are such that, if all the members of $u$ satisfy $\phi$, then $f^{\prime} u$ satisfies $\phi$ and is not a member of $u$. Whenever these two conditions are satisfied for all values of $u$, one or other of two conclusions follows: namely, either (1) $\phi ! x$ is not a predicative property; or (2), if $\phi ! x$ is predicative and defines the class $w$, then there must be no such entity as $f^{\prime} w$. This is proved very simply as follows:-If there is such a class as $w$, and such an entity as $f^{\prime} w$, then, since every member of $w$ satisfies $\phi$, it follows that $f^{\prime} w$ satisfies $\phi$; but, conversely, $f^{\prime} w$ must be not a member of $w$, and must therefore not have the property $\phi$, since $w$ consists of all terms having the property $\phi$. In the special case of the ordinals, our two alternatives are : (1) the ordinals do not form a class; (2) although they form a class, they have no ordinal number. The second alternative is

"The inverted comma may be read "of." The notation $f^{\prime} u$ means the same as $f(u)$, but is for several reasons more convenient. 
equivalent to the assumption that either the whole series of ordinals is not well ordered, or, if it is well ordered, the dual property " $\alpha$ and $\beta$ are ordinal numbers, and $\alpha$ is less than $\beta$ " is non-predicative; so that the series as a whole has no definite type, i.e., no ordinal number. The supposition that the whole series of ordinals is not well ordered can be disproved*; hence we are left with the alternatives that either (1) the property " $x$ is an ordinal number" is non-predicative, or (2), though " $x$ is an ordinal number" is predicative, " $x$ and $y$ are ordinal numbers and $x$ is less than $y$ " is non-predicative.

We have seen that Burali-Forti's contradiction is a particular case of the following:-

"Given a property $\phi$ and a function $f$, such that, if $\phi$ belongs to all the members of $u, f^{\prime} u$ always exists, has the property $\phi$, and is not a member of $u$; then the supposition that there is a class $w$ of all terms having the property $\phi$ and that $f^{\prime} w$ exists leads to the conclusion that $f^{\prime} w$ both has and has not the property $\phi . "$

This generalization is important, because it covers all the contradictions that have hitherto emerged in this subject. In the case of the class of terms which are not members of themselves, we put " $x$ is not a member of $x$ " for $\phi ! x$, and $u$ itself for $f^{\prime} u$. In this case, owing to the fact that $f^{\prime} u$ is $u$ itself, we have only one possibility: namely, that " $x$ is not a member of $x$ " is non-predicative. In other cases, we have two possibilities, and it may often be difficult to decide which of them to choose.

When we have a pair such as $\phi$ and $f$ above, we can define, in terms of $f$ alone, without introducing $\phi$, a series ordinally similar to that of all ordinals, and obtain, as regards this series, a contradiction analogous to Burali-Forti's, provided $f$ satisfies certain conditions. We do this as follows :-Taking any class $x$, for which $f^{\prime} x$ exists, take $f^{\prime} x$ as the first term of our series, take the $f$ of the class got by adding $f^{\prime} x$ to $x$ as the second term, and so on. Generally, the successor of any term is the $f$ of the class consisting of that term together with all its predecessors and $x$, and the successor of a class $u$ of terms having no maximum is the $f$ of the class consisting of the whole segment defined by the class $u$. This gives Cantor's two principles of formation, and we can define the property of occurring in this series by

\footnotetext{
* This supposition can be disproved (by the generalized form of induction which applies throughout any well-ordered series) by means of the theorem that every segment of the series of ordinals is well ordered. It is not disproved by Jourdain's theorem, that every series which is not well ordered must contain a part of type * $\omega$; for this theorem depends upon Zermelo's axiom, of which the truth is doubtful.
} 
the generalized form of induction.* We may then, subject to certain conditions as to $f$, substitute for $\phi$ the property of occurring in the $f$-series starting from $x$. If $f$ has the property that, if $u$ is composed of terms of the above series, then $f^{\prime} u$ exists and is not a member of $u$, it will follow that the whole series does not form a class; for, if it did, its $f$ would both be and not be a member of the series. In the particular case of the ordinals, if $u$ is a class of ordinals, $f^{\prime} u$ is their immediate successor ; the whole series of ordinals can be generated by the above method, starting from 0 . In the case of " $x$ is not an $x$," $f^{\prime} x$ is $x$ itself : if we start from any class which is not a member of itself, and proceed by the above method, we obtain a series, like the series of all ordinals, $\uparrow$ consisting entirely of classes which are not members of themselves, and the series as a whole does not form a class.

The above considerations point to the conclusion that the contradictions result from the fact that, according to current logical assumptions, there are what we may call self-reproductive processes and classes. That is, there are some properties such that, given any class of terms all having such a property, we can always define a new term also having the property in question. Hence we can never collect all the terms having the said property into a whole; because, whenever we hope we have them all, the collection which we have immediately proceeds to generate a new term also having the said property. It is probable, in view of the above general form for all known contradictions, that, if $\phi$ is any demonstrably non-predicative property, we can actually construct a series, ordinally similar to the series of all ordinals, composed entirely of terms having the property $\phi$. Hence, if the terms satisfying $\phi$ can be arranged in a series ordinally similar to a segment of the series of ordinals, it follows that no contradiction results from assuming that $\phi$ is a predicative property. But this proposition is of very little use, until we know how far the series of ordinals goes; and at present it is not easy to see where this series begins to be non-existent, if such a bull may be permitted.

* This is done as follows :-A property is inductive in the $f$-series if whenever it belongs to a class $u$ it belongs to the class got by adding $f^{\prime} u$ to $u$, and whenever it belongs to each of a set of classes it belongs to their logical sum, i.e., to the class of members of members of the set. A term belongs to the " $f$-series starting from $x$ " if it possesses every property which is possessed by $x$ and is inductive in the $f$-series.

$+I$ owe the proof that this series is well ordered and ordinally similar to the series of all ordinals to Mr. G. G. Berry, of the Bodleian Library. 
II.

We have now seen the nature of the contradictions which beset the theory of the transfinite: we have seen that they are not an isolated fow, but can be manufactured in any required number by a recipe; we have seen that all of them belong to a certain definite type, and we have seen that none of them are essentially arithmetical, but all belong to logic, and are to be solved, therefore, by some change in current logical assumptions. I propose, in this section, to consider three different directions in which such a change may be attempted. I shall endeavour to set forth the advantages and disadvantages of each of the three, without deciding in favour of any one of them.

What is demonstrated by the contradictions we have considered is broadly this: "A propositional function of one variable does not always determine a class." *

In view of this fact, it is open to us, prima facie, to adopt one or other of two theories. We may decide that all ordinary straightforward propositional functions of one variable determine classes, and that what is needed is some principle by which we can exclude the complicated cases in which there is no class. In this view, the state of things is like that in the differential calculus, where every commonplace continuous function has a derivative, and only rather complicated and recondite functions have to be excluded. The other theory which suggests itself is that there are no such things as classes and relations and functions as entities, and that the habit of talking of them is merely a convenient abbreviation.

The first of these two theories itself divides into two, according as we hold that what classes have to avoid is excessive size, or a certain characteristic which we may call zigzagginess. Of these, the second is the more conservative, i.e., it preserves more of the theory of the transfinite than the first. Both preserve more of it than does the theory that there are no such things as classes. I shall consider these three theories in the following order, and by the following names:-
A. The zigzag theory.
B. The theory of limitation of size.
C. The no classes theory.

\footnotetext{
* Here it is to be understood that the arguments which show that there is not always a class also show that there is not always a separable entity which is the propositional function (as opposed to its value) ; also that, some propositional functions of two variables do not determine a relation either in intension or in extension, if we mean by a relatiun a separable entity which can be considered apart from related terms.
} 


\section{A. The Zigzag Theory.}

Each of the three theories can be recommended as plausible by the help of certain a priori logical considerations. In the zigzag theory, we start from the suggestion that propositional functions determine classes when they are fairly simple, and only fail to do so when they are complicated and recondite. If this is the case, it cannot be bigness that makes a class go wrong; for such propositional functions as " $x$ is not a man" have an exemplary simplicity, and are vet satisfied by all but a finite number of entities. In this theory, as well as in the theory of limitation of size, we define a predicative propositional function as one which determines a class (or a relation, if it contains two variables); thus in the zigzag theory the negation of a predicative function is always predicative. In other words, given any class $u$, all the terms which are not members of $u$ form a class which may be called the class not-u.

If now $\phi ! x$ is a non-predicative function, it follows that, given any class $u$, there must either be members of $u$ for which $\phi ! x$ is false, or members of not- $u$ for which $\phi ! x$ is true. (For, if not, $\phi ! x$ would be true when, and only when, $x$ is a member of $u$; so that $\phi ! x$ would be predicative.) It thus appears that $\phi ! x$ fails to be predicative just as much by the terms it does not include as by the terms it does. Again, given any class $u$, the property $\phi ! x$ belongs either to some, but not all, of the members of $u$, or to some, but not all, of the members of not- $u$. This is the zigzag property which gives its name to the theory we are considering. This theory is specially suggested by the argument of Cantor's proof that there is no greatest cardinal. This proof, as we have already seen, constructs a would-be class $w$ by the norm " $x$ is not a member of the class with which it is correlated by the relation $R$," where $R$ is a relation which correlates individuals with classes. Such would-be classes, as we saw, are very apt to be not classes, and they all have a certain zigzag quality, in the fact that $x$ is a $w$ when $x$ is not a member of its correlate, and is not a $w$ when $x$ is a member of its correlate.

The full development of this theory requires axioms as to the kinds of functions that are predicative. It has the great advantage that it admits as predicative all functions which can be stated simply, and only excludes such complicated cases as might well be supposed to have strange properties.*

The principal objection to this theory, so far as it is at present de-

- For suggestions of a solution more or less on the above lines, see my Principles of Mathematics, $\$ \oint 103,104$. 
veloped, is that the axioms as to what functions are predicative have to be exceedingly complicated, and cannot be recommended by any intrinsic plausibility. This is a defect which might be remedied by greater ingenuity, or by the help of some hitherto unnoticed distinction. But hitherto, in attempting to set up axioms for this theory, I have found no guiding principle except the avoidance of contradictions; and this, by itself, is a very insufficient principle, since it leaves us always exposed to the risk that further deductions will elicit contradictions. The general postulate, that predicative propositional functions must have a certain simplicity, does not lend itself readily to the decision whether this or that propositional function has the requisite degree of simplicity. Nevertheless, since these difficulties are all such as further research might conceivably remove, the theory is not to be rejected wholly, but is rather to be retained as one of those that are possible. Speaking broadly, one may say that it applies better to cardinal than to ordinal contradictions: it deals more readily with such difficulties as that of the class of classes which are not members of themselves than with such difficulties as that of BuraliForti.

The zigzag theory, in some form or other, is that assumed in the definitions of cardinal and ordinal numbers as classes of classes (if numbers are supposed to be entities). For all these classes of classes, if they are legitimate, must contain as many members as there are entities altogether; hence, if bigness makes classes go wrong, as we suppose in the "limitation of size" theory, cardinals and ordinals so defined will be illegitimate classes. Dr. Hobson has various criticisms on these definitions of cardinals and ordinals; but on the zigzag theory his criticisms can, I think, be all satisfactorily met.

Dr. Hobson says": "It has been seen that the assumptions that an ordered aggregate possesses a definite order type and a definite cardinal number, which can be treated as objects, lead to the contradiction pointed out by Burali-Forti." This statement seems to me somewhat too sweeping. It is quite open to us to hold every ordered aggregate possesses a definite cardinal number, and that every ordered aggregate which is ordinally similar to a segment of the ordinals in order of magnitude possesses a definite ordinal number. All that Burali-Forti's contradiction forces us to admit is that there is no maximum ordinal, i.e., that the function " $\alpha$ and $\beta$ are ordinal numbers, and $\alpha$ is less than $\beta$ " and all other functions ordinally similar to this one are non-predicative. In the same way the difficulty of the greatest cardinal is met by denying that the 
defining functions of Cantor's omitted classes are predicative in certain cases. Thus we conclude that in this theory there is a greatest cardinal, but there is no greatest ordinal; in each case contradictions are avoided by regarding certain functions as not predicative.

Dr. Hobson distinguishes two methods of establishing the existence of a class of mathematical entities : the genetic method and the method by postulation. He rejects the former, as regards cardinals and ordinals; but he seems not to perceive that this can only be done by recognizing that there may be no class even where there is a perfectly definite norm. From his No. 2 one would suppose that he regards the norm as a sufficient condition for the class; yet, later on, he refuses to admit classes which are defined by unimpeachable norms. It seems hardly correct to say, as he does: "In the genetic method, as applied to the construction of the whole series of ordinal members, this notion of correspondence plays no part." (No. 6, p. 177.) It is the notion of correspondence which defines the class of relations constituting an ordinal number; this class consists of all the relations which are like* a certain given relation. "The existence of a number," he truly says, "is constantly inferred from that of a single unique ordered aggregate." (Ib.) But there can be no objection to this procedure, unless on the ground that, when $P$ is given, " $Q$ is like $P$ " is not predicative in respect of $Q$.

It is, of course, very easy to prove, when we have one series of a certain type, that there are an infinite number of series of the same type. To do this we need only substitute other terms for the terms of our series. Suppose, e.g., our series is composed of numbers. We may substitute Socrates for any terms of our series; this will give as many new series of the same type as there are terms in the given series. If our series is infinite, we can obtain $\boldsymbol{N}_{0}$ series of the same type by merely knocking off terms at the beginning; and so on. Thus, if multiplicity of series of a given type is desired, there is no difficulty in obtaining as many series of the given type as there are entities altogether, i.e., the maximum cardinal number of series of the given type. (For, instead of Socrates, we may substitute any other term not occurring in our series.) Thus it is not the case that the genetic method involves "the setting up of a scale of standard.s, to which standards no eggregates not consisting of the preceding num hors conform" (No. 6, p. 173), though I do not see what harm there wouid be if this were the case.

- I use like to mean ordinally similar to. For the precise deinition cf. Principles of N5athematics, $\$ 253$. 
The same remark applies to the criticism (No. 7, p. 179) of the definition of a cardinal as a class of similar classes.* It is very easy to show that the number of classes similar to the class of numbers from 0 to $n$ is as great as the total number of entities; and, even if no other class were similar to this class, that would not, so far as I can see, constitute any objection. The number $n+1$ would, in that case, be the class whose only member is the class of numbers from 0 to $n$.

Dr. Hobson explains that his opinion and mine are at variance as to the definition of cardinals because I, unlike him, "regard the activities of the mind as irrelevant in questions of existence of entities" (No. 7, p. 180). This is a philosophical difference, and, like all philosophical differences, it ought not to be allowed to affect the detail of mathematics, but only the interpretation. Mathematics would be in a bad way if it could not proceed until the dispute between idealism and realism has been settled. When a new entity is introduced, Dr. Hobson regards the entity as created by the activity of the mind, while I regard it as merely discerned; but this difference of interpretation can hardly affect the question whether the introduction of the entity is legitimate or not, which is the only question with which mathematics, as opposed to philosophy, is concerned.

There is another passage in No 7 (p. 179) which calls for explanations, namely, the following:- "Russell objects to the conception of a number as the common characteristic of a family of equivalent aggregates on the ground that there is no reason to think that such a single entity exists with which the aggregates have a special relation, but that there may be many such entities. The mind does, however, in point of fact, in the case of finite aggregates at least, recognize the existence of such single entity, the number of the aggregates; and this is a valid result of our mental activity, subject to the law of contradiction."

In the first place, it is not merely the case that "there may be many such entities," but that there demonstrably are as many as there are entities altogether. Given any many-one relation having the property that when, and only when, $u$ and $v$ are similar classes, there is an entity $a$ to which both $u$ and $v$ have the relation $S$, the converse domain of $S$ (i.e., the terms to which classes have the relation $S$ ) will have all the formal properties of cardinal numbers. $t$ Now, if there is one such

- This definition is due to Frege. See his Grundlagen der Arithmetik, Breslau, 1S84, pp. 79, 85.

t For a development of this point of view see $\$ 2$ of "La Logique des Relations," Revwe de Mathématiques, Vol. vur. 
relation as $S$, it is very easy to prove that there are as many as there are entities altogether; and, if there is no such relation as $S$, then there are no such entities as cardinal numbers. (There might be ${ }^{-c a r d i n a l}$ numbers for some classes and not for others, if there was a relation such as $S$ which had some classes in its domain, but no relation such as $S$ which had all classes in its domain.)

The supposition that there is no such relation as $S$ is disproved by the fact that the relation of a class to the class of all classes similar to it has the properties we wish $S$ to have. This disproof is rejected by Dr. Hobson, since be considers that it involves improper classes. His position seems to be that, at least in the case of finite aggregates, "the mind" immediately recognizes a certain relation of the sort required. The simplest formal statement of this point of view is, roughly, as follows :-

In beginning cardinal arithmetic we introduce a new indefinable $S$, concerning which we lay down the indemonstrable properties : *

(1) $S$ is a many-one relation ;

(2) Every finite class (and, presumably, some infinite classes) have the relation $S$ to some term ;

(3) When, and only when, two finite classes (and, presumably, some pairs of infinite classes) are similar they both have the relation $S$ to the same term;

(4) Things which are not classes do not have the relation $S$ to anything.

The reason that $S$ has to be indefinable and the above propositions indemonstrable is that, if we regard the above propositions as giving a definition of $S$ "by postulates," they do not determine $S$, since an infinite number of relations (if any) fulfil the above conditions, and every entity will, for a suitahle $S$, be the cardinal number (in respect of that $S$ ) of some class which has a cardinal number. Moreover, the recognition by "the mind," which Dr. Hobson speaks of, is precisely the process of introducing an indefinable. It is a process of which, in certain cases, I fully recognize the validity and the necessity; but indeñnables and indemonstrables are to be diminished in number as much as possible.t Moreover, in the case supposed, where Dr. Hobson says

* It is probably possible to simplify the statement of these indemonstrables.

+ This is merely the truism with which Dedekind begins "Was sind und was sollen die Zahien," namely: "Was beweisbar ist, soll in der Wissensohaft nicht ohne Beweis geglaubt werảen." 
that "the mind" recognizes such entities, I am unable to agree: if he said "my mind," I should have taken his word for it; but, personally, I do not perceive such entities as cardinal numbers, unless as classes of similar classes.

\section{B. The Theory of Limitation of Size.}

This theory is naturally suggested by the consideration of BuraliForti's contradiction, as well as by certain general arguments tending to show that there is not (as in the zigzag theory) such a thing as the class of all entities. This theory naturally becomes particularized into the theory that a proper class must always be capable of being arranged in a well-ordered series ordinally similar to a segment of the series of ordinals in order of magnitude; this particular limitation being chosen so as to advoid Burali-Forti's contradiction.* We still have the distinction of predicative and non-predicative functions; but the test of predicativeness is no longer simplicity of form, but is a certain limitation of size. In this theory, if $u$ is a class, " $x$ is not a member of $u$ " is always non-predicative; thus there is no such class as not $u$.

The reasons recommending this view are, roughly, the following :-We saw, in the first part of this paper, that there are a number of processes, of which the generation of ordinals is one, which seem essentially incapable of terminating, although each process is such that the class of all terms generated by it (or a function of this class) ought to be the last term generated by that process. Thus it is natural to suppose that the terms generated by such a process do not form a class. And, if so, it seems also natural to suppose that any aggregate embracing all the terms generated by one of these processes cannot form a class. Consequently there will be (so to speak) a certain limit of size which no class can reach; and any supposed class which reaches or surpasses this limit is an improper class, i.e., is a non-entity. The existence of selfreproductive processes of this kind seems to make the notion of a totality of all entities an impossible one; and this tends to discredit the zigzag theory, which admits the class of all entities as a valid class.

This theory has, at first sight, a great plausibility and simplicity, and I am not prepared to deny that it is the true solution. But the plausibility and simplicity tend rather to disappear on examination.

Let us first recall the generalizations of Burali-F'orti's contra-

- This view has been. adrocated by Jourdain, "On the Transfinite Cardinal Numbers of" Well-ordered Aggregates," No. 4, Phil. Mag., January, 1904; also "On Transfinite Cardinal Numbers of the Exponential Form," Phil. Mag., January, 1905. 
diction which we obtained in the first part of this paper. The fundamental proposition is: "Given a property $\phi$ and a function $f$ such that, if $\phi$ belongs to all the members of any class $u$, then $f^{\prime} u$ always exists and has the property $\phi$, but is not a member of $u$, it follows that either $\phi$ is non-predicative or, if $\phi$ is predicative and determines the class $w$, then $f^{\prime} w$ does not exist."

The theory of limitation of size neglects the second alternative (that $f^{\prime} w$ may not exist), and decides for the first (that $\phi$ is not predicative). Thus, in the case of the series of ordinals, the second alternative is that the whole series of ordinals has no ordinal number, which is equivalent to denying the predicativeness of " $\alpha$ and $\beta$ are ordinal numbers, and $\alpha$ is less than $\beta$." The adoption of this alternative would enable us to hold that all ordinals do form a class, and yet there is no greatest ordinal. But the theory in question rejects this alternative, and decides that the ordinals do not form a class. The only case in which this is the only alternative is when $f^{\prime} u$ is $u$ itself; otherwise we always have a choice.

A great difficulty of this theory is that it does not tell us how far up the series of ordinals it is legitimate to go. It might happen that $\omega$ was already illegitimate: in that case all proper classes would be finite. For, in that case, a series ordinally similar to a segment of the series of ordinals would necessarily be a finite series. Or it might happen that $\omega^{2}$ was illegitimate, or $\omega^{\omega}$ or $\omega_{1}$ or any other ordinal having no immediate predecessor. We need further axioms before we can tell where the series begins to be illegitimate. For, in order that an ordinal $a$ may be legitimate, it is necessary that the propositional function " $\beta$ and $\gamma$ are ordinal numbers less than $a$, and $\beta$ less than $\gamma$ " should be predicative. (Here, of course, "less than $a$ " must be replaced by some property not involving $a$, but such that, if $a$ is legitimate, the property is equivalent to being less than $a$.) But our general principle does not tell us under what circumstances such a function is predicative.

It is no doubt intended by those who advocate this theory that all ordinals should be admitted which can be defined, so to speak, from below, i.e., without introducing the notion of the whole series of ordinals. Thus they would admit all Cantor's ordinals, and they would only avoid admitting the maximum ordinal. But it is not easy to see how to state such a limitation precisely: at least, I have not succeeded in doing so. The merits of this theory, therefore, would seem to be less than they at first appear to be. 


\section{The No Classes Theory.}

In this theory classes and relations are banished altogether.* It is not necessary to the theory to assume that no functions determine classes and relations; all that is essential to the theory is to abstain from assuming the opposite. This is the strong point of the theory we are now to consider: the theory is constituted merely by abstinence from a doubtful assumption, and thus whatever of mathematics it permits us to obtain is indubitable in a way which anything involving classes or relations cannot be. The objections to the theory are (1) that it seems obvious to common sense that there are classes; (2) that a great part of Cantor's theory of the transfinite, including much that it is hard to doubt, is, so far as can be seen, invalid if there are no classes or relations ; (3) that the working out of the theory is very complicated, and is on this account likely to contain errors, the removal of which would, for aught we know, render the theory inadequate to yield the results even of elementary arithmetic.

To explain fully how this theory is to be developed would take too much space. Some of its main points may, however, be briefly set forth.

Instead of a function $\phi ! x$, where the notation inevitably suggests the existence of something denoted by " $\phi$," we proceed as follows:Let $p$ be any proposition, and $a$ a constituent of $p$. (TVe may say broadly that $a$ is a constituent of $p$ if $a$ is mentioned in stating $p$.) Then let " $p \frac{x}{a}$ " denote what $p$ becomes when $x$ is substituted for $a$ in the place or places where $a$ occurs in $p$. For different values of $x$ this will give us what we have been accustomed to call different values of a propositional function. In place of $\phi$ we have now two variables, $p$ and $a$ : in respect to the different values of $p \frac{x}{a}$, we may call $p$ the prototype and $a$ the origin or initial subject. (For a may be taken as being, in a generalized sense, the subject of $p$.) Consider now such a statement as " $p \frac{x}{a}$ is true for all values of $x$." Let $b$ be an entity which is not a constituent of $p$, and put $q=p \frac{b}{a}$; then " $q \frac{x}{b}$ is true for all values of $x$ " is

* It must be understood that the postulate of the existence of classes and relations is exposed to the same arguments, pro and con., as the existence of propositional functions as separable entities distinct from all their values. Thus, in the theory we are considering, anything said about a propositional function is to be regarded as a mere abbreviation for a statement about some or all of its values. 
equivalent to " $p \frac{x}{a}$ is true for all values of $x$." Thus, subject to a certain reservation, the statement " $p \frac{x}{a}$ is true for all values of $x$ " is independent of the initial subject $a$, and thus may be said to depend only upon the form of $p .{ }^{*}$ Statements of this sort replace what would otherwise be statements having propositional functions for their arguments. For example, instead of " $\phi$ is a unit function" (i.e., "There is one, and only one, $x$ for which $\phi ! x$ is true"), we shall have "There is an entity $b$ such that $p \frac{x}{a}$ is true when, and only when, $x$ is identical with $b . "$ There will not now be any such entity as the number 1 in isolation; but we shall be able to define what we mean by "One, and only one, proposition of the type $p \frac{x}{a}$ (for a given $p$ and $a$ ) is true." Instead of saying "The class $u$ is a class which has only one member," we shall say (as above) "There is an entity $b$ such that $p \frac{x}{a}$ is true when, and only when, $x$ is identical with $b$." Here the values of $x$ for which $p \frac{x}{a}$ is true replace the class $u$; but we do not assume that these values collectively form a single entity which is the class composed of them.

There is not much difficulty in re-wording most definitions so as to fit in with the new point of view. But now the existence theorems become hard to prove. We can manufacture enough different propositions to show what is now equivalent to the existence of $\omega$ and $\aleph_{0}$, though the process is cumbrous and artificial. We shall be able, by continuing a similar process, to prove the existence of various transfinite ordinal types. But we shall not be able to prove the existence of all the usual ordinal types. I do not know at what point the series begins to be non-existent; but I cannot at present, in this method, prove the existence of $\omega_{1}$ or $\kappa_{1}$, which must therefore be considered for the moment as undemonstrated.

I hope in future to work out this theory to the point where it will appear exactly how much of mathematics it preserves, and how much it forces us to abandon. It seems fairly clear that ordinary arithmetic, analysis, and geometry, and, indeed, whatever does not involve the later

* The reserration is merely that the initial subject must not occur in the prototype except in the places which we wish to be variable. For example, if our prototype is " $3>2$," and our initial nubject is 3 , the substitution of $x$ for 3 gives " $x>2$." But, if we now take 2 as our initial subject, so that our prototype is " $2>2$," the substitution of $x$ gives " $x>x$," which is not the propositional function we want. 
transfinite numbers, can be stated, though in a roundabout and difficult way, without the use of classes and relations as independent entities. A certain amount, also, of transfinite arithmetic can be preserved; but it is not easy to discover how much. The theory is safe, but drastic; and, if, in fact, there are classes and relations, it is unnecessarily difficult and complicated. For the present, therefore, it may be accepted as one way of avoiding contradictions, though not necessarily the way.

III.

I come now to the second of our difficulties, namely, the doubt as to the truth of Zermelo's axiom. This is dealt with by Dr. Hobson in his Nos. 10 and 11, with which I find myself in complete agreement.*

All that I wish to do is to state the question in various forms, and to point out some of its bearings. I shall assume the existence of classes and relations, for the sake of simplicity of statement. The difficulty is of a different kind, and is more easily apprehended by this form of statement.

Zermelo's axiom asserts the possibility of picking out one from each of the classes contained in a given class (excepting the null class). It has hitherto been commonly assumed by mathematicians, and Zermelo has the merit of explicitly mentioning the assumption. The axiom may be stated as follows:- "Given any class $w$, there is a function $f^{\prime} u$ such that, if $u$ is an existent† class contained in $w$, then $f^{\prime} u$ is a member of $u$." That is, the axiom asserts that we can find some rule by which to pick out one term from each existent class contained in $w$. The axiom may also be stated: "Given a set $k$ of all existent classes contained in a certain class $w$, there is a many-one relation $R$, whose domain is $k$, which is such that, if $u$ is a member of $k$, the term to which $u$ has the relation $R$ is a member of $u . "$ The axiom can be stated in a form which does not involve classes, functions, or relations, but I shall not give this form of statement, as its complication makes it almost unintelligible.

A simple illustration may serve to show the nature of the difficulty as regards this axiom, and to introduce the analogous "multiplicative axiom." Given $N_{0}$ pairs of boots, let it be required to prove that the number of boots is even. This will be the case if all the boots can be divided into

- Though I do not agree with his special criticism of Mr. G. H. Hardy in No. 12, according to which the second figure in Mr. Hardy's sequences "would have indefinitely great values for all numbers $\beta$ of the second class, and thus that for sufficiently great ordinal numbers of the second class the corresponding sequences can have no existence."

$\dagger$ An existent class is a class having at least one member. 
two classes which are mutually similar. If now each pair has the right and left boots different, we need only put all the right boots in one class, and all the left boots in another: the class of right boots is similar to the class of left boots, and our problem is solved. But, if the right and left hoots in each pair are indistinguishable, we cannot discover any property belonging to exactly half the boots. Hence we cannot divide the boots into two equal parts, and we cannot prove that the number of them is even. If the number of pairs were finite, we could simply choose one out of each pair ; but re cannot choose one out of each of an infinite number of pairs unless we have a rule of choice, and in the present case no rule can be found.

The problem involved in the above illustration raises grave difficulties in regard to many elementary theorems about multiplication of cardinals. Multiplication has been defined as follows by Mr. A. N. Whitehead :-*

Let $k$ be a set of classes no two of which have any common terms. Then we define the "multiplicative class of $k$ " (denoted by $\times$ ' $k$ ) as the class formed by picking one and only one term out of each class belonging to $k$, and doing this in all possible ways. That is, one member of $\times$ ' $k$ is a class consisting of one member of each class belonging to $k$. Then the number of terms in $\times$ ' $k$ is defined to be the product of the numbers of the various classes belonging to $k$. This definition is perfectly satisfactory when the number of classes which are members of $k$ is finite, and also when each class which is a member of $k$ has some peculiar term (for example, if each is given as a well-ordered series, and we can pick out the first term). But in other cases it is not obvious that there is any rule hy which we can pick out just one term of each member of $k$, and therefore it is not obvious that $x^{\prime} k$ has any members at all. Hence, as far as the definition shows, the product of an infinite number of factors none (f) which is zero might be zero. Thus, in the case of the boots, we wished to pick out one boot from each pair, but we could find no rule by which this was to be done.

What is required is not that we should actually be able to pick out one term from each class which is a member of $k$, but that there should be (whether we can specify it or not) at least one class composed of one term from each member of $k$. It there is one, there must be many, unless all the members of $k$ are unit classes; for, given one such class, if $u$ is a member of $k$, and $x$ is the member of $u$ which is picked out, we can substitute for $x$ any other member of $u$-say $y$-and we still have a nember of $\times$ ' $k$. Thus the axiom we need may be stated: "Given a 
mutually exclusive set of classes $k$, no one of which is null, there is at least one class composed of one term out of each member of $k$."

This axiom is more special than Zermelo's axiom. It can be deduced from Zermelo's axiom ; but the converse deduction, though it may turn out to be possible, has not yet, so far as I know, been effected. I shall call this the multiplicative axiom.

The multiplicative axiom has been employed constantly in proofs of theorems concerning transfinite numbers. It is open to everybody, as yet, to accept it as a self-evident truth, but it remains possible that it may turn out to be capable of disproof by reductio ad absurdum. It mav also, of course, be capable of proof, but that is far less probable. A class of classes of which this axiom holds may conveniently be called a multipliable class of classes.

The above axiom is required for identifying the two definitions of the finite. We may define a finite cardinal number

(a) As a cardinal number which obeys mathematical induction starting from 0 ;

(b) As a cardinal number such that any class which has that number contains no part similar to itself.

We will for the present call any number of the kind (a) an inductive number, and any number of the kind $(b)$ a finite number. Then it is easy to prove that all inductive numbers are finite; that every class whose number is infinite contains a part whose number is $N_{0}$ (where $N_{0}$ is defined as the number of inductive numbers), and vice versa; and that, if the number of classes contained in a finite class is always finite, then all finite numbers are inductive numbers. But, so far as I know, we cannot prove that the number of classes contained in a finite class is always finite, or that every finite number is an inductive number.*

The multiplicative axiom is also required for proving that the number of terms in $\alpha$ sets of $\beta$ terms is $\alpha \times \beta$, i.e., for connecting addition and multiplication. We cannot even prove, without this axiom, that the number of terms in $\alpha$ sets of $\beta$ terms is always the same. Similarly, we cannot prove that the product of $a$ factors each equal to $\beta$ is $\beta^{a}$ (taking

- Burali-Forti has shown that the two definitions of the finite can be identified if we assume the following axiom:- "If $u$ is any class of existent classes, the number of members of $u$ is less than or equal to the number of members of members of "l." ("Le Classi finite," Proceedings of the Accademia Reale delle Scienze di Torino, 1896-7.) This axiom leads at once to the result that the number of classes contained in a finite class must be finite, whence the conclusion follows, as above indicated. The axiom as it stands is untrue : it is necessary to assume that the classes are mutually exclusive, or something analogous. Whether it will then give the desired result I do not know.

SER. 2. VOL. 4 . No. 915 . 
Cantor's definition of exponentiation), ${ }^{*}$ or even that it is always the same number.

And in the case of the $N_{0}$ pairs of boots we cannot prove that the number of boots is $N_{0}$ (i.e., $N_{0}+N_{0}$ ), except in the case where we can distinguish right and left boots.

The existence of $\times$ ' $k$ can be proved whenever any method exists of picking out one term from each member of $k$. If, for example, all terms which are members of members of $k$ belong to some one well-ordered series, we get a member of $x^{\prime} k$ by picking out the first terms of the various members of $k$ ( $k$ being assumed to be a set of mutually exclusive existent classes). It does not follow that $\times{ }^{\prime} k$ exists when every member of $k$ can be well ordered : for there will always be many ways of well ordering each member of $k$, and we need some rule for picking out one, in each case, of the various possible ways of well ordering each member. That is, we need a term of the multiplicative class of the class of which a single member is the class of relations by which a single member of $k$ is well ordered.

If $k$ is any set of mutually exclusive existent classes, and if we form another class $k^{\prime}$ by substituting for every member $u$ of $k$ the class $\left(u^{\prime}\right)$ of all existent classes contained in $u$, then $k^{\prime}$ is a set of mutually exclusive existent classes, and $\times^{\prime} k^{\prime}$ exists, since $k$ is a member of $X^{\prime} k^{\prime}$ (because each $u$ is a member of its $u^{\prime}$ ).

Assuming that $k$ is a set of mutually exclusive existent classes, there are certain cases in which the existence of $\times$ ' $k$ can be proved, because there is some structure which enables us to pick out particular terms from members of $k$. Such, for example, is the following case :Suppose there is some one-many relation $P$, such that each term of $k$ consists of all the terms to which some term of the domain of $P$ has the relation $P$, and suppose further that every term of the domain of $P$ has the relation $P$ to itself : then the domain of $P$ is a member of the multiplicative class of $k$.

If $k$ is a set of mutually exclusive existent classes, $\times$ ' $k$ exists when, and only when, there is a one-one relation $S$ whose domain is $k$, and which relates each class $u$, belonging to $k$, to a member of $u$; for, when this condition is satisfied, the converse domain of $S$ is a member of $\times ' k$; and, given a term of $\times$ ' $k$, the relation of members of $k$ to the corresponding members of the given term of $\times{ }^{\prime} k$ is an $S$ fulfilling the above conditions. Another way of stating the same thing is that $\times$ ' $k$ exiets when, and only when, there is some function $f^{\prime} u$ such that, 
if $u$ is a member of $k, f^{\prime} u$ is a member of $u$; for the $f^{\prime} \mathrm{s}$ of the various members of $k$ malie up a term of $k$. A sufficient condition for the existence of $\times$ ' $k$ whenever $k$ is a set of mutually exclusive existent classes is that there should exist a function $f^{\prime} u$ such that, if $u$ is any existent class, $f^{\prime} u$ is a member of $u$. This is equivalent to Zermelo's axiom, ${ }^{*}$ and it is not, so far as I know, a necessary condition for the existence of $\times$ ' $\%$ in all such cases.

Zermelo's axiom is a generalized form of the multiplicative axiom, and is interesting because he has shown + that, if it is true, then every class can be well ordered. Since it is doubtful whether all classes obey Zermelo's axiom, we may define a Zermelo class as one which does obey the axiom; that is, $w$ is a Zermelo class if there is at least one many-one relation $R$ such that the domain of $R$ consists of all existent classes contained in $w$, and if $u$ has the relation $R$ to $x$, then $x$ is $a$ member of $u$. That is, a class $w$ is a Zermelo class if there is a method of correlating each existent class contained in $w$ with one of its members. Zermelo proves that any class $w$ for which this holds can be well ordered. The converse is obvious; for, if $w$ is well ordered, we correlate each existent class $u$, contained in $v$, with the first term of $u$, which gives a relation $R$ satisfying the above conditions. Hence Zermelo's axiom holds of those classes which can be well ordered, and of no others.

By applying his axiom to the class of all entities, we find that, if it holds universally, there must be a function $f^{\prime} u$ such that, if $u$ is any existent class, then $f^{\prime} u$ is a member of $u$. Conversely, if there is such a function, Zermelo's axiom is obviously always satisfied. Hence, if there is a class of all entities, his axiom is equivalent to: "There is a function $f^{\prime} u$ such that, if $u$ is any existent class, $f$ " $u$ is a member of $u$."

I think that Zermelo's axiom, applied in its functional form, and without the assumption that there are classes or relations, leads to the result that any propositional function only satisfied by terms of one type is such that all the terms satisfying it can be well ordered. If it should appear, on other grounds, that this is not always true, it would follow that Zermelo's axiom, in its functional form, is false. Whether or not it is true in the form in which it applies only to classes is a question which requires for its answer a previous decision as to what propositional functions are predicative: the more we restrict the notion of class the more likely this form of Zermelo's axiom is to be true, and the less

* Assuming that there is a class of all entities. But, if there is no such class, we only have to adopt the statement of Zermelo's axiom which does not assume that there are classes.

† Math. Ann., Vol. LIx., pp. 514-516. 
information it gives us. To discover the conditions subject to which Zermelo's axiom and the multiplicative axiom hold would be a very important contribution to mathematics and logic, and ought not to be beyond the powers of mathematicians.

It is easy to see that, if $v$ is any Zermelo class, and $k$ is a set of mutually exclusive existent classes which between them contain all the terms $v$ and no more, then $k$ is a multipliable class of classes. For every member of $k$ is an existent class contained in $v$; hence, if we pick out one term from each existent class contained in $v$, we incidentally pick out one term from each member of $k$. Thus the universal truth of Zermelo's axiom involves the universal truth of the multiplicative axiom. The converse, so far as I know, has not been proved, and may or may not be true.

It should be observed that, both in the case of Zermelo's axiom and in that of the multiplicative axiom, what we are primarily in doubt about is the existence of a norm or property such as will pick out one term from each of our aggregates; the doubt as to the existence of a class which will make this selection is derivative from the doubt as to the existence of a norm.

The problem concerned in such cases is like that of the "lawless" decimal, which reduces to the problem of the "lawless" class of finite integers. If we consider all the classes that can be formed of finite integers, it seems at first sight obvious that many will consist of a perfectly haphazard collection, not definable by any iormula. But this is open to doubt. It would seem that, as Dr. Hobson urges, an infinite aggregate requires a norm, and that such haphazard collections as seem conceivable are really non-entities. In the case of Cantor's "proof" that there are more classes of finite numbers than there are finite numbers, it is shown that no one denumerable set of formulæ will cover all classes of finite numbers; but the class shown to be left over in each case is defined by a formula in the process of showing that it is left over. Thus this process gives no ground for thinking that there are classes of finite numbers which are not definable by a formula.

To sum up: there are two analogous axioms-Zermelo's and the multiplicative axiom-which have been habitually employed by mathematicians in reasonings about the transfinite, but which, most likely, are not true without some restriction. Without them, we cannot, so far as at present appears, identify the two definitions of the finite, or establish the usual relations of addition, multiplication, and exponentiation. If 'Zermelo's axiom were true, every class would be well ordered, and also, I think, every aggregate of terms possessing some property. But in this 
respect the problem considered in our second part is dependent upon that considered in our first part.

The general position advocated in the foregoing paper may be briefly stated as follows :-

When we say that a number of objects all have a certain property, we naturally suppose that the property is a definite object, which can be considered apart from any or all of the objects which have, or may be supposed to have, the property in question. We also naturally suppose that the objects which have the property form a class, and that the class is in some sense a new single entity, distinct, in general, from each member of the class. Both these natural suppositions can be proved, by arguments so short and simple that they scarcely admit a possibility of error, to be at any rate not universally true. We may, in view of this fact, adopt one of two courses: we may either decide that the assumptions in question are always false, or endeavour to find conditions subject to which they are true, these conditions being such as to exclude the cases where the falsehood of the assumptions can be proved. The latter course has the advantage of being more consistent with common sense, and of preserving more of Cantor's work; but it has, as yet, the disadvantage of great uncertainty and artificiality in detail, owing to the absence of any broad principle by which to decide as to which functions are predicative. The former course, in practice, merely involves abstaining from the doubtful assumptions, and does not commit us to the view that they are false; it is therefore, so long as any doubt subsists, the prudent plan to pursue the former course as far as possible. It appears on examination that, without supposing either of the suspected assumptions to be ever true, we can construct ordinary mathematics and most of the theory of the transfinite; and in this development we meet with no contradictions, so far as is known at present. Whether it is possible to rescue more of Cantor's work must probably remain doubtful until the fundamental logical notions employed are more thoroughly understood. And whether, in particular, Zermelo's axiom is true or false is a question which, while more fundamental matters are in doubt, is very likely to remain unanswered. The complete solution of our difficulties, we may surmise, is more likely to come from clearer notions in logic than from the technical advance of mathematics; but until the solution is found we cannot be sure how much of mathematics it will leave intact.

[Note added February 5th, 1906.-From further investigation I now feel hardly any doubt that the no-classes theory affords the complete solution of all the difficulties stated in the first section of this paper.] 